\title{
No recovery of replication-competent HIV-1 from human liver macrophages
}

\author{
Abraham J. Kandathil, ${ }^{1}$ Sho Sugawara, ${ }^{1}$ Ashish Goyal, ${ }^{2}$ Christine M. Durand, ${ }^{1}$ Jeffrey Quinn, ${ }^{1}$ Jaiprasath Sachithanandham, ${ }^{1}$ \\ Andrew M. Cameron, ${ }^{3}$ Justin R. Bailey, ${ }^{1}$ Alan S. Perelson, ${ }^{2}$ and Ashwin Balagopal ${ }^{1}$ \\ 'Department of Medicine, Johns Hopkins University, Baltimore, Maryland, USA. ${ }^{2}$ Theoretical Biology and Biophysics, Los Alamos National Laboratory, Los Alamos, New Mexico, USA. ${ }^{3}$ Department of Surgery, \\ Johns Hopkins University, Baltimore, Maryland, USA.
}

\begin{abstract}
Long-lived HIV-1 reservoirs that persist despite antiretroviral therapy (ART) are a major impediment to a cure for HIV-1. We examined whether human liver macrophages (LMs), the largest tissue macrophage population, comprise an HIV-1 reservoir. We purified LMs from liver explants and included treatment with a T cell immunotoxin to reduce T cells to $1 \%$ or less. LMs were purified from 9 HIV-1-infected persons, 8 of whom were on ART (range 8-140 months). Purified LMs were stimulated ex vivo and supernatants from 6 of 8 LMs from persons on ART transmitted infection. However, HIV-1 propagation from LMs was not sustained except in LMs from 1 person taking ART for less than 1 year. Bulk liver sequences matched LM-derived HIV-1 in 5 individuals. Additional in vitro experiments undertaken to quantify the decay of HIV-1-infected LMs from 3 healthy controls showed evidence of infection and viral release for prolonged durations ( $>170$ days). Released HIV-1 propagated robustly in target cells, demonstrating that viral outgrowth was observable using our methods. The $t_{1 / 2}$ of HIV-1-infected LMs ranged from 3.8-55 days. These findings suggest that while HIV-1 persists in LMs during ART, it does so in forms that are inert, suggesting that they are defective or restricted with regard to propagation.
\end{abstract}

\section{Introduction}

HIV-1 cure is a global health priority. Although an HIV-1 cure is possible, there are multiple challenges that hinder the design of a durable cure (1). Long-lived cellular reservoirs of HIV-1 exist in all patients, thwarting HIV-1 eradication. A long-lived cellular reservoir is defined as one that persists despite the suppression of HIV-1 replication that occurs with combination antiretroviral therapy (ART), and from which infectious virus can be recovered ex vivo to perpetuate new transmission (2), modeling the natural history of infection after ART interruption. The best-characterized HIV-1 cellular reservoir is the resting memory $\mathrm{CD}^{+} \mathrm{T}$ cell latent reservoir, but other reservoirs may exist (3).

Tissue macrophages are abundant, long-lived, and susceptible to HIV-1 infection; however, their role as a long-lived HIV-1 cellular reservoir has been difficult to address in humans. There has been recent interest in understanding the role of tissue resident macrophages in HIV-1 (4-6). We undertook a proof-ofprinciple study to address whether human liver macrophages (LMs), comprising up to $90 \%$ of all tissue macrophages (7), harbor infectious HIV-1 during ART.

\section{Results}

Purity of isolated LMs. We first developed a protocol to purify LMs that limited the inadvertent presence of $\mathrm{CD} 4^{+} \mathrm{T}$ cells that could confound studies of HIV-1 reservoirs (8). To detect T cell contamination in LMs, we used a sensitive qPCR assay for CD3 $\varepsilon$ mRNA

Conflict of interest: The authors have declared that no conflict of interest exists. Submitted: April 23, 2018; Accepted: July 26, 2018

Reference information: J Clin Invest. 2018;128(10):4501-4509.

https://doi.org/10.1172/JCl121678.
(Supplemental Figure 1A; supplemental material available online with this article; https://oi.org/10.1172/JCI121678DS1). The assay identified total $\mathrm{T}$ cell contamination in macrophages at a ratio of between 1:100 and 1:1,000, confirming $99 \%$ to $99.9 \%$ purity (Supplemental Figure 1B). The $C D 3 \varepsilon$ qPCR assay circumvented the requirement of flow cytometry or FACS to ensure purity, which would have been challenging given limited cell numbers. To enhance LM purity, we progressively depleted $\mathrm{T}$ cells (Figure 1A), employing a strategy of positive selection of $\mathrm{T}$ cells using CD3 microbeads, plastic adherence, and deprivation of $\mathrm{T}$ cell mitogens over more than 30 days. $T$ cell depletion concluded with the use of Resimmune, a high-affinity anti-CD3 recombinant diphtheria toxin that has been used in patients to deplete $\mathrm{T}$ cells (9): we confirmed that Resimmune was specific for T cells, not macrophages (Supplemental Figure 1, C and D). In vitro we observed over $80 \%$ reduction in $\mathrm{T}$ cell viability using Resimmune (Supplemental Figure 1C) (10). The multistep protocol resulted in little evidence of $\mathrm{T}$ cell contamination in purified LMs (Figure 1B). In addition, purified LMs were cultured for more than 30 days in the presence of antiretrovirals (tenofovir disoproxil fumarate [TDF], emtricitabine [FTC], and raltegravir [RTG]) to fully suppress ex vivo infection of LMs by dying or phagocytosed CD $4^{+} \mathrm{T}$ cells that would have confounded our results.

Next, we tested whether HIV-1-infected $\mathrm{CD} 4^{+} \mathrm{T}$ cells might falsely enhance the apparent abundance of macrophage infection if the 2 cell populations were cocultured to model the possibility of T cells contaminating LMs at the limit of our detection. We cocultured different combinations of HIV-1-infected versus uninfected $\mathrm{CD}^{+}{ }^{+} \mathrm{T}$ cells with HIV-1-infected or uninfected monocyte-derived macrophages (MDMs) in the presence or absence of Resimmune, mimicking possible culture conditions (Supplemental Figure 2). 

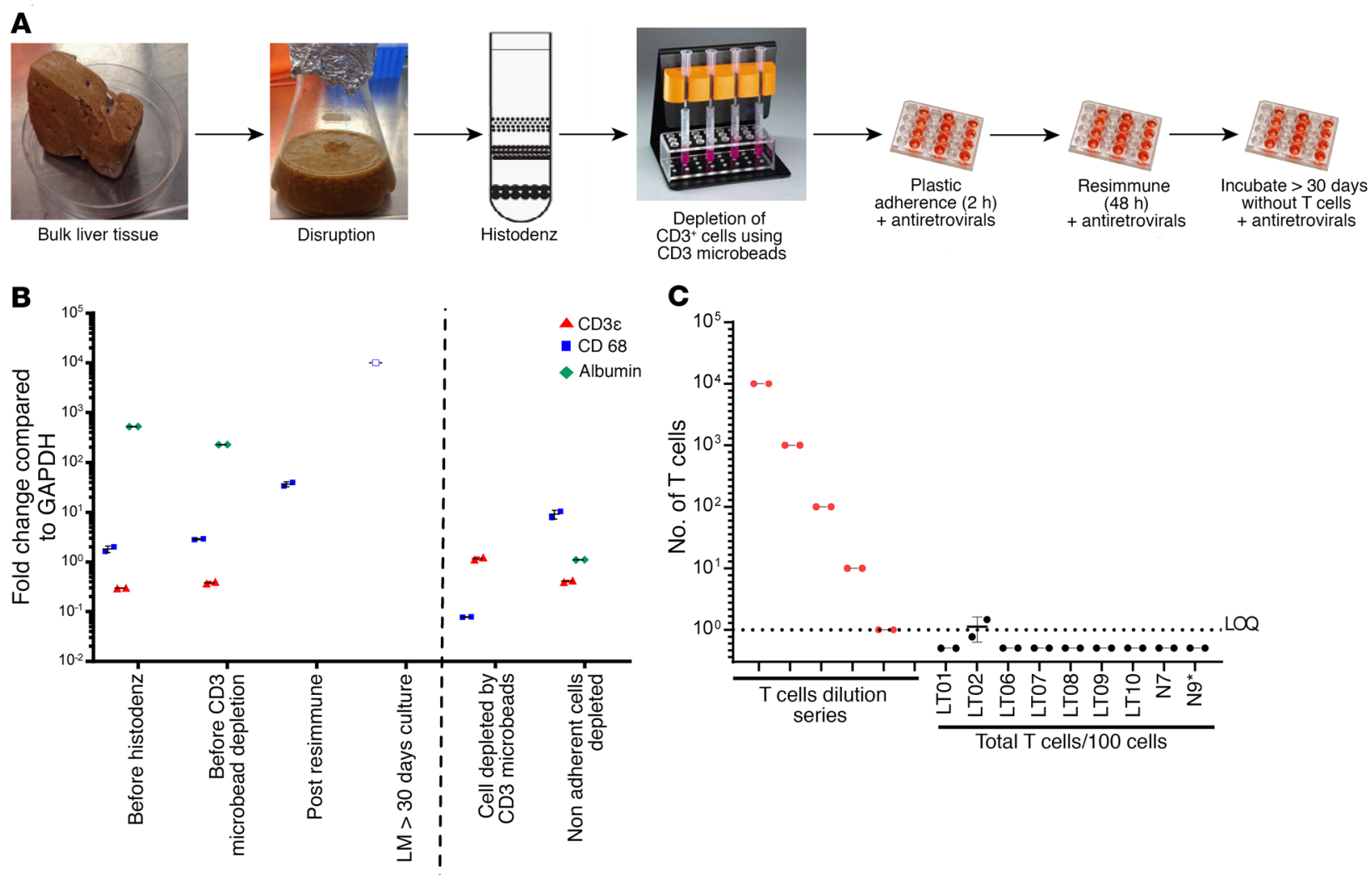

Figure 1. Purification of liver macrophages yields minimal T cell contamination. (A) LMs were purified from fresh liver tissue using a multistep protocol that progressively depletes T cells: bulk liver tissue was disrupted, centrifuged on a Histodenz gradient (Sigma-Aldrich), and followed by depletion of T cells using CD3 microbeads. Mononuclear cell fractions were incubated on plastic to separate adherent LMs from nonadherent cells and then incubated with Resimmune, a high-affinity T cell toxin, to deplete remaining T cells. LMs were incubated for more than 30 days, deprived of T cell mitogens, and maintained in the presence of antiretrovirals (ARVs) to prevent ex vivo infection. (B) The purification protocol depletes T cells. In a sample isolation of LMs from an HIV-1 uninfected liver, a sensitive qPCR assay for CD3\& mRNA showed the absence of detectable T cells. LMs and hepatocytes were also detected by qPCR assays for CD68 and albumin mRNA, respectively. The fold increases were compared with GAPDH. However, when GAPDH was not detected, as shown by the open blue box (CD68), fold-change was calculated by assigning GAPDH the last cycle number of the QPCR. (C) LM purification resulted in minimal T cell contamination in tissues taken from HIV-1-infected people. LM purity and T cell contamination were measured in duplicate on LMs isolated from 9 HIV-1-infected people before Resimmune and compared with a dilution series of total T cells. The protocol resulted in no detectable T cell contamination of LMs from 8 of 9 participants. In LMs from LTO2, 1\% T cell contamination was detectable, which was removed by maintaining the LMs in culture devoid of T cell mitogens for more than 90 days and treatment with Resimmune. The T cell dilution series represents the standard curve derived by performing qPCR for CD3\& mRNA on total RNA extracted from 10 -fold dilutions of unactivated $\mathrm{CD}^{+} \mathrm{T}$ cells isolated by MACS separation from PBMCs obtained from a healthy donor. Error bars indicate mean $\pm \mathrm{SD}$. ${ }^{*}$ For $\mathrm{N} 9$, there appeared to be PCR inhibition in the sample as indicated by poor detection of a housekeeping gene by qPCR.

Cells were infected separately and then combined, testing whether quantities of HIV-1 proviral DNA were different between test conditions after 30 days of coculture. $\mathrm{CD}^{+} \mathrm{T}$ cells were mixed with macrophages in a ratio of 1:100, conservatively reflecting the lower limit of detection of our CD3E mRNA assay for T cell contamination. Upon mixing, cocultured cells were incubated with antiretrovirals to prevent transmission of infection from one cell population to the other. After 30 days of coculture of MDMs from 3 healthy donors with $\mathrm{CD} 4^{+} \mathrm{T}$ cells from the same donors, we did not find evidence that $\mathrm{CD} 4^{+} \mathrm{T}$ cell infection enhanced the measured abundance of HIV-1 proviral DNA in mixed cultures. Moreover, MDMs showed evidence of infection even in the presence of contaminating $\mathrm{CD}^{+} \mathrm{T}$ cells that were never infected. Since antiretrovirals were introduced into the wells at the time of coculture, the last result demonstrates that macrophage infection was sufficient on its own to yield proviral DNA 30 days after infection, irrespective of whether $\mathrm{CD}^{+} \mathrm{T}$ cells contaminated the cultures.
Intriguingly, however, we found that only 2 of 3 donors showed evidence of HIV-1 infection in isolated MDMs that were never cocultured with $\mathrm{CD} 4^{+} \mathrm{T}$ cells after 30 days. In parallel, we characterized the donors' CCR5 432 status: donor 1 had a copy of the CCR5 32 mutation, whereas donors 2 and 3 had only WT alleles. Taken together, these results exclude the possibility that $\mathrm{CD}^{+}$ $\mathrm{T}$ cells could confound our findings in LMs from HIV-1-infected people. Furthermore, we were able to detect $C D 3 \varepsilon$ mRNA in lysed cells more than 30 days after coculture (data not shown), indicating that our assays were sensitive enough to detect the presence of contaminating $\mathrm{T}$ cells if they were present.

Liver macrophage viral outgrowth assay. We used a variety of cell lines to identify which would be the most robust at replicating HIV-1 from LMs (Supplemental Figure 3). Curiously, although CEMx174 cells have been previously described to not contain CCR5 on their surface, their replication of R5-tropic HIV-1 was comparable to CCR5-expressing MOLT-4 cells that have been 


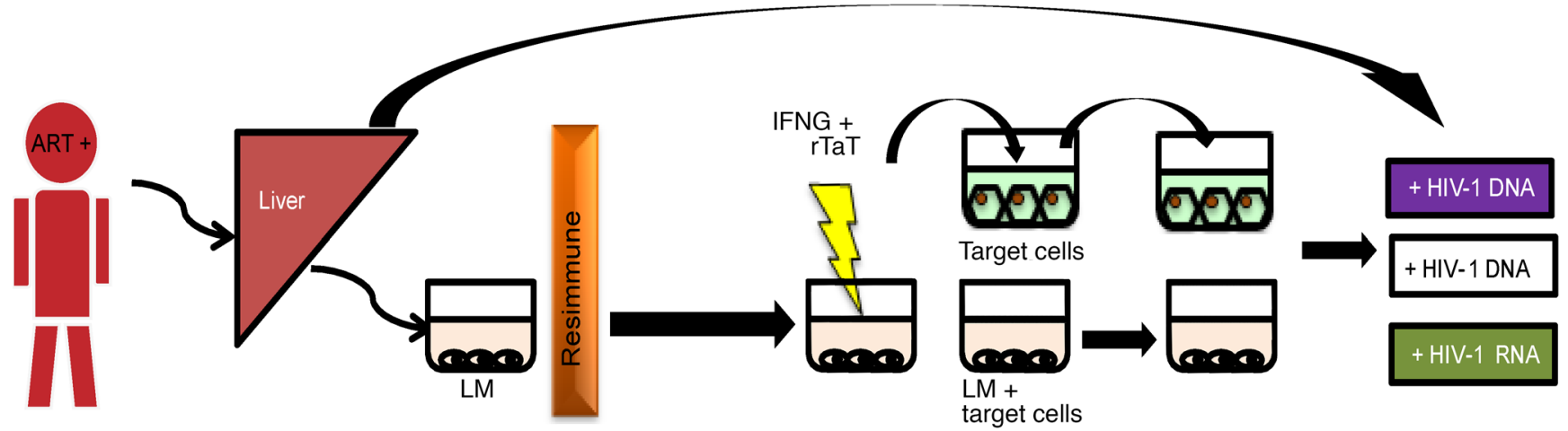

Figure 2. Schema for VOA performed on purified LMs. After purification of primary human LMs from HIV-1-infected subjects, cells were maintained ex vivo for more than 30 days. The VOA was performed on LMs from individuals taking ART. Isolated LMs were treated with Resimmune over 48 hours to deplete any remaining T cells. Following stimulation with IFNG and HIV-1 rTat, LM supernatants were filtered and transferred to target cells twice over 15 days and incubated. In addition, target cells were added to LMs. Cell-associated and supernatant HIV-1 DNA and RNA levels were measured on day 11 in target cells.

recently described (11). In addition, CEMx174 cells gave the most reproducible results when inoculated with $\mathrm{R} 5$-tropic HIV1 , as has been described recently for macrophage-derived SIV (12). Next, we infected LMs in vitro using a bicistronic GFPexpressing R5-tropic strain of HIV-1 that has been previously shown to replicate robustly in human MDMs (13). We continued to detect HIV-1 RNA in LM supernatants for more than 100 days after infection despite periodic media change, strongly supporting productive infection and indicating that these were viruses that were released by LMs. Although the amounts of HIV-1 RNA released from infected LMs were lower than has been described for $\mathrm{CD}^{+} \mathrm{T}$ cells, they are consistent with previous reports of macrophage infection $(4,5)$. The LM supernatants were then transferred to CEMx174 cells, which began to fluoresce green after incubation (Supplemental Figure 4). Moreover, HIV-1 RNA was detectable in CEMx174 supernatants for up to 15 days despite media changes (Supplemental Figure 5).

To mimic recovery of LM-derived HIV-1 from ARTsuppressed individuals, HIV-1-infected LMs were treated with antiretrovirals that were sufficient to inhibit infection. After 21 days of antiretroviral exposure, LMs were stimulated with interferon gamma (IFNG) followed by recombinant Tat (rTat) and their supernatants were transferred to CEMx174, testing whether HIV-1 released from LMs that themselves could not complete the HIV-1 life cycle (because of antiretrovirals) was sufficient to propagate infection in a viral outgrowth assay (VOA). Upon lysis, CEMx174cells were found to contain HIV-1 proviral DNA using a sensitive qPCR assay (Supplemental Figure 6), whereas control CEMx174 cells that were inoculated with media from uninfected LMs failed to show HIV-1 DNA. Additionally, CEMx174 cells that were inoculated with LMs treated with antiretroviral for 12 and 20 days propagated HIV1 for up to 15 days after incubation (Supplemental Figure 5). Taken together, these results support the assumption that if LMs are productively infected, CEMx174 target cells can be used to propagate infectious virus in an adapted VOA.

LMs from HIV-1-infected people. To test the contribution of LMs to HIV-1 infection in vivo, we purified LMs from liver tissue of HIV-1infected individuals. Fresh liver tissue was obtained from a deceased HIV-1-infected patient (N7) who had not been taking ART for more than 6 months and who had a plasma HIV-1 RNA level greater than
$500,000 \mathrm{cp} / \mathrm{ml}$. Following isolation and culture of LMs that contained no detectable $\mathrm{T}$ cells, we detected polyadenylated HIV-1 RNA that is found only in spliced and genomic RNA (14) in supernatants 18 days after purification, demonstrating that LMs can be infected with HIV-1 in vivo. We next examined whether infectious viruses were recoverable from LMs in HIV-1-infected people who were virologically suppressed on ART. Fresh liver explant tissues were obtained intraoperatively from 7 HIV-1-infected individuals undergoing liver transplantation and from 1 individual at the time of death (N9). These individuals were taking ART at the time of liver recovery and had undetectable plasma HIV-1 RNA levels. Total HIV-1 proviral DNA was detectable in low levels in bulk liver tissue from 7 of 8 participants (data not shown). A median of $5.5 \times 10^{5} \mathrm{LMs}$ (range $4.8 \times 10^{5}-1.4 \times 10^{6}$ ) were purified from all participants and $\mathrm{T}$ cell exclusion was confirmed in all but 1 subject (LTO2) who had approximately $1 \mathrm{~T}$ cell in $100 \mathrm{LMs}$ (99\% pure) (Figure 1C).

IFNG+rTat was used to stimulate LMs from HIV-1-infected people more than 30 days after LM purification (Figure 2). In one instance (N9), bacterial contamination was noted early after LM purification; therefore, LMs were stimulated only after 6 days after isolation. Following stimulation of LMs, supernatants were filtered and transferred twice over a 15-day interval to CEMx174 cells. Proviral DNA was detected in target cells incubated with LM supernatants from 6 of 8 participants and never in negative control target cells that were incubated with media alone (Figure $3 \mathrm{~A}$ and Table 1). However, with the exception of LTO2, target cells from other isolated LMs did not show evidence of HIV-1 propagation by way of HIV-1 RNA (Table 1). We did not observe high-level replication, as has been seen from resting CD4 ${ }^{+}$T cells (11). LTO2 was also distinct in having taken ART for the shortest duration prior to liver transplantation (Figure 3B and Table 1). Taken together, these results demonstrate that LMs can harbor HIV-1 for prolonged periods, but these are inert with respect to propagation unless sampling occurred very early after ART-mediated suppression. We confirmed the presence of replication-competent HIV- 1 in resting $\mathrm{CD} 4^{+} \mathrm{T}$ cells in this cohort. Using the conventional quantitative VOA that has been previously described and used extensively to quantify the latent reservoir (15), we estimated a similarly sized reservoir in resting CD4 ${ }^{+} \mathrm{T}$ cells from 2 of the people who also had contemporaneous LM VOA performed (Supplemental Table 1). 


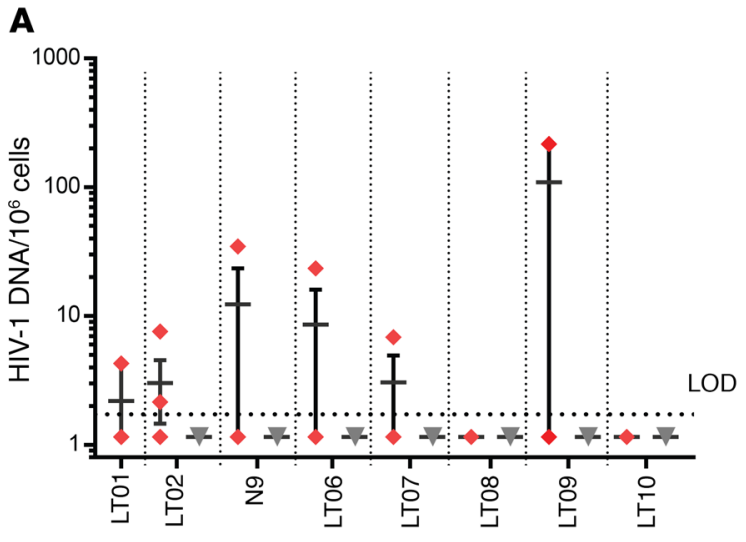

B

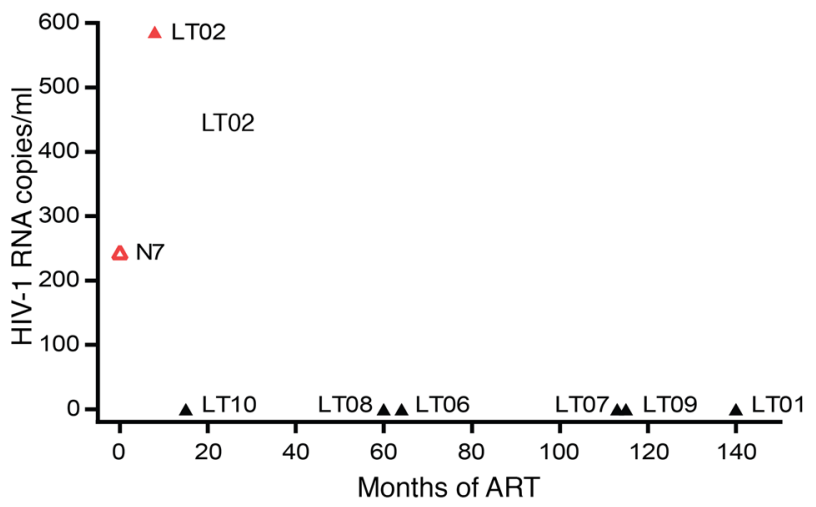

Figure 3. HIV-1 transmission from LMs of HIV-1-infected individuals. (A) LM VOA results for all HIV-1-infected individuals. Using qPCR, HIV-1 proviral DNA was measured in lysed target cells that were incubated with LM supernatants from each donor (red diamonds). Error bars represent mean \pm SEM for some with multiple positive values. Grey triangles indicate negative control target cells that were inoculated with media alone before Iysis. N7 is not included in this figure because HIV-1 RNA was detectable in LM supernatants before initiation of the LM VOA. (B) LM VOA yields HIV-1 RNA only in people with ART exposure less than 1 year. HIV-1 RNA was measured in target cell supernatants in LM VOA from all people, and in N7 LM supernatants directly. HIV-1 RNA was only detectable from LMs in 2 people who were also those who had the least amount of exposure to ART before liver explantation. $\mathrm{N} 9$ is not included in this figure because the duration of ART prior to liver explantation was not available. LOD, limit of detection; ND, not done.

We were not able to differentiate between unintegrated and integrated DNA present in the LMs before VOA because of the limited number of LMs available for sequencing. We were only able to amplify a 160-bp portion of the HIV-1 pol gene from LM VOA of 5 individuals. These sequences closely matched bulk liver sequences, sharing a median $p$ distance of 0.012 (range $0-0.031$ ) with their respective bulk liver tissue HIV-1 sequences; in contrast, the median interindividual $p$ distance was 0.037 (range $0.023-0.068, P<0.005$ ), despite limited variability in this region of the pol gene (nucleotide position 4900-5061 in reference HXB2 genome) (Figure 4).

HIV-1-infected LMs persist variably in vitro. Previous estimates of the $t_{1 / 2}$ of HIV-1-infected macrophages (14 days) have been relatively short compared with resting $\mathrm{CD} 4^{+} \mathrm{T}$ cells (44 months) (16). We attempted to measure the longevity of HIV-1-infected LMs using the LMs that we had previously infected in vitro. We observed minimal cell death within the first 4 weeks of infection. HIV-1 RNA remained detectable in LM supernatants from all 3 participants for greater than 30 days of infection (doi), and thereafter gradually decayed (Figure $5, \mathrm{~A}-\mathrm{C}$ ). Our findings were similar in LMs infected with the R5-tropic BaL HIV-1 strain (Supplemental Figure 7). LMs that were lysed on 181 doi revealed intracellular HIV-1 RNA. HIV-1 RNA from donor 1 LMs was largely undetectable in supernatants by 100 doi but showed occasional blips, whereas HIV-1 RNA was still detectable in LM supernatants from donors 2 and 3. HIV-1 RNA release continued even when antiretrovirals (TDF/FTC/RTG) were added to LM media in concentrations that were sufficient to inhibit replication in $\mathrm{CD} 4^{+} \mathrm{T}$ cells (Figure 5D). We observed an inconstant pattern with regard to HIV-1 dynamics in LMs during the 24 days of antiretrovirals, whereas $\mathrm{GFP}^{+}$cells were visible throughout antiretroviral incubation (data not shown). Based on the viral kinetics during ART, the median of the infected LMs $t_{1 / 2}$ was estimated at 6.8 days (range 3.8-55 days) (Figure 5, A-C), although in some wells persistent HIV-1 RNA release prevented accurate calculation of $t_{1 / 2}$. It should be noted that the $t_{1 / 2}$ was calculated based on decay kinetics of released RNA and this could possibly be longer because of the existence of virus-containing compartments in LMs (17). Indeed, we observed abundant intracellular HIV-1 RNA in LMs even in the absence of concomitant release of HIV-1 RNA in the supernatant (Supplemental Figure 7), consistent with this phenomenon.

\section{Discussion}

In this study we demonstrate, using an adapted VOA and negligible $\mathrm{T}$ cell contamination, that freshly isolated LMs from HIV-1-infected individuals harbor HIV-1 for prolonged durations, despite longstanding suppressive ART. We also show, in contrast to previous estimates, that LMs persist despite HIV-1 infection and that they release virions for months in vitro. However, despite these findings, we did not find sufficient evidence that LMs contained replication-competent virus, nor could we induce these viruses to propagate robustly.

HIV-1 reservoirs persist for extended durations during ART and lead to virologic rebound when treatment is interrupted. Previously, tissue macrophages were thought to have short half-lives, and were disqualified as candidate reservoirs. Our understanding of the longevity of tissue macrophages, however, has undergone a marked revision: macrophages seed peripheral tissues during embryonic development and replenish themselves locally (18-23). LMs have been estimated to live for approximately 14 months in an animal model (24), were reported to contain HIV-1 in situ in the pre-ART era, and support HIV-1 replication in vitro (25-29). Therefore, it is logical to hypothesize that LMs contain HIV-1 reservoirs. Surprisingly, we found evidence for HIV-1 in LMs from a person who took suppressive ART for up to 11.7 years (Figure 3B).

Despite our findings, we found limited evidence of exponential HIV-1 propagation from LMs, or of latent LM infection, in contrast to resting $\mathrm{CD} 4^{+} \mathrm{T}$ cell reservoirs. In only one case did purified LMs transmit infection to target cells that resulted in HIV-1 RNA release (LTO2), but this person was only on ART for 8 months prior to liver explantation and also had evidence of low-level $\mathrm{T}$ cell contamination (Figure 3B). We conjectured that there may be several reasons why we did not find evidence of replication-competent HIV-1 in LMs. First, SAMHD1, an HIV-1 
Table 1. Liver macrophage viral outgrowth assay results

$\begin{array}{lcccc}\text { ID } & \begin{array}{c}\text { ART duration, } \\ \text { months }\end{array} & \begin{array}{c}\text { Target cells used } \\ \text { in VOA } \\ \text { Activated T cells }\end{array} & \begin{array}{c}\text { VOA results } \\ \text { RNA }\end{array} \\ \text { LT01 } & 140 & - & + \\ \text { LTO2 }^{A} & 8 & \text { CEMx174 } & + & + \\ \text { N7 } & - & \text { ND } & \text { ND } & \text { ND } \\ \text { N9 } & \text { NA } & \text { CEMx174 } & - & + \\ \text { LT06 } & 64 & \text { CEMx174 } & - & + \\ \text { LT07 } & >113 & \text { CEMx174 } & - & + \\ \text { LT08 } & >60 & \text { CEMx174 } & - & - \\ \text { LT09B } & 115 & \text { CEMx174 } & - & + \\ \text { LT10 } & 15 & \text { CEMx174 } & - & -\end{array}$

Duration of uninterrupted ART exposure is listed when documentation was available (no exposure for N7, no clear documentation aside from the presence of ART for N9). Plasma HIV-1 RNA was undetectable for all subjects at the time of explantation, except for N7. Results of the LM VOA for each person are shown. ${ }^{A}$ LTO2 was an elite suppressor with a pre-ART plasma HIV-1 RNA level of $74 \mathrm{cp} / \mathrm{ml}$. BLTO9 was a retransplant, so the explant was never exposed to active plasma viremia in vivo.

restriction factor that has been well-described in macrophages, may promote more faulty reverse transcription events, rendering proviral DNAs defective in macrophages where the gene is most active (30). Second, LMs may contain additional restriction factors, such as the newly described MARCH8 (31), that dampen the infectivity of virions that are released from macrophages. Third, LMs with replication-competent HIV-1 may be more likely to be cleared by adaptive responses than macrophages with defective HIV-1, similar to what has been described in resting $\mathrm{CD} 4^{+} \mathrm{T}$ cells (32). Fourth, HIV-1-infected LMs may fully decay within the first year after ART initiation, reflecting the intrinsic $t_{1 / 2}$ that others and we have observed (5). It is worth noting here that the median $t_{1 / 2}$ of LMs that we observed was 6.8 days, which is higher than recent estimates. Finally, it is possible that we did not find evidence of replication-competent HIV-1 in LMs because we found remarkable heterogeneity in the $t_{1 / 2}$ estimates of LMs, even within the same donor.

Several groups have reported that retroviruses persist in tissue macrophages in animal models (33), mostly focusing on SIV infection of macaques and the central nervous system $(12,34)$. Our data are most consistent with those reported recently by Honeycutt et al. from studies with myeloid-only humanized mice, which demonstrated recovery of infectious virus from mice that were infected with HIV-1 and then treated with ART for 3 months or less (5). Infectious virus was only recovered in the minority of cases, however, suggesting that HIV-1-infected myeloid cells might decay fully during prolonged ART (5). In comparison, there has been little human data from tissue macrophages.

Our study had several limitations. Prior studies of tissue macrophages and HIV-1 (such as those using in situ hybridization) were limited by the amount of tissue used to test for macrophage reservoirs (25). Although in our study we tested larger numbers of purified, viable LMs from multiple individuals, we were still limited in the total numbers of LMs that we studied in any one experiment $\left(\leq 1.4 \times 10^{6}\right.$ cells). In addition, the overall size of our study was small, depending largely on liver explants obtained during liver transplantation in HIV-1-infected people, an infrequent event ( $0.4 \%$ of all liver transplantations in the United States). Indeed, the number of people studied depended on procuring liver tissue from nearly every liver transplantation for an HIV-1-infected organ recipient at our institution from July, 2013, until July, 2016 $(n=7$; Table 1). Although indirect evidence of $\mathrm{T}$ cell phagocytosis by macrophages has been recently reported (8), our findings did not appear to be affected by phagocytosis. We used a highly sensitive and well-validated qPCR assay for T cells that only identified CD3 $\varepsilon$ mRNA in LMs from 1 of 9 people (Supplemental Figure 1). We also performed in vitro coculture experiments showing that when HIV-1-infected T cells were cocultured with MDMs, we did not observe evidence of enhancement in HIV-1 proviral DNA despite 30 days of coculture (Supplemental Figure 2). We found that HIV-1-infected LMs in vitro transmitted infection to CEMx174 cells and showed evidence of replication in the absence of $\mathrm{CD} 4^{+}$ T cells (Supplemental Figures 4 and 5). It is still possible, however, that LM phagocytosis of HIV-1-infected $\mathrm{CD}^{+} \mathrm{T}$ cells occurred in vivo, prior to liver explantation, although distinguishing that possibility from the absence of phagocytosis may not have clinical relevance. A possible limitation to our study is the presence of contaminating resting $\mathrm{CD} 4^{+} \mathrm{T}$ cells in our LM cultures that were lower in abundance than our ability to detect them; however, we note that the likelihood of infection of the resting memory $\mathrm{CD} 4^{+} \mathrm{T}$ cell fraction is incredibly small (35). Thus, it is virtually impossible for contamination of $1 \%$ or less to have confounded our findings $(P<0.01)$. Because of the inflammatory nature of liver disease, which resulted in liver transplantation for many of our subjects, a further limitation is in distinguishing LMs that may have arisen recently from inflammatory monocytes, from sessile liver macrophages that are likely to have resided in the liver for a much longer period (21). Accordingly, we defined the cells in our study as LMs, rather than subsetting them by their origin. We also had difficulty obtaining full-length HIV-1 sequences from our LM viral outgrowth, indicative of the low abundance of infection. Whereas the sequences that we found matched sequences from bulk liver tissue (Figure 4), supporting a common origin, we were unable to identify major defects in LM-derived virus, nor were we able to confirm its R5-tropism because an insufficient template limited our ability to sequence longer fragments of the HIV-1 genome.

In conclusion, we recovered HIV-1 from LMs that were purified from HIV-1-infected people taking suppressive ART. However, we did not find evidence that HIV-1 in LMs was replication competent, nor could we induce HIV-1 to propagate from LMs.

\section{Methods}

Liver tissue. Whole liver tissues from HIV-1-infected individuals $(n=9)$ were obtained from 2 sources (Supplemental Table 2). The National Disease Research Interchange (NDRI) provided fresh tissues (N7 and N9) from HIV-1-infected individuals at the time of their demise. Fresh liver tissues that were intended for discard were also procured from HIV-1-infected patients (LT01, LTO2, LT06, LT07, LT08, LT09, and LT10) undergoing liver transplantation at Johns Hopkins Hospital (JHH). Tissues were obtained intraoperatively at the time of explantation and processed immediately for isolation of LMs. 
A

\begin{tabular}{|c|c|c|c|c|c|c|}
\hline \multicolumn{2}{|c|}{} & \multicolumn{5}{|c|}{ LM } \\
\cline { 2 - 7 } & N7 & LT01 & LT02 & LT06 & LT09 \\
\hline \multirow{7}{*}{} & N7 & 0 & 0.068 & 0.37 & 0.025 & 0.31 \\
\cline { 2 - 7 } & LT01 & 0.068 & 0 & 0.068 & 0.045 & 0.023 \\
\cline { 2 - 7 } & LT02 & 0.037 & 0.68 & 0.012 & 0.025 & 0.025 \\
\cline { 2 - 7 } & LT06 & 0.031 & 0.045 & 0.037 & 0.019 & 0.025 \\
\cline { 2 - 7 } & LT09 & 0.025 & 0.068 & 0.043 & 0.025 & 0.031 \\
\hline
\end{tabular}

B

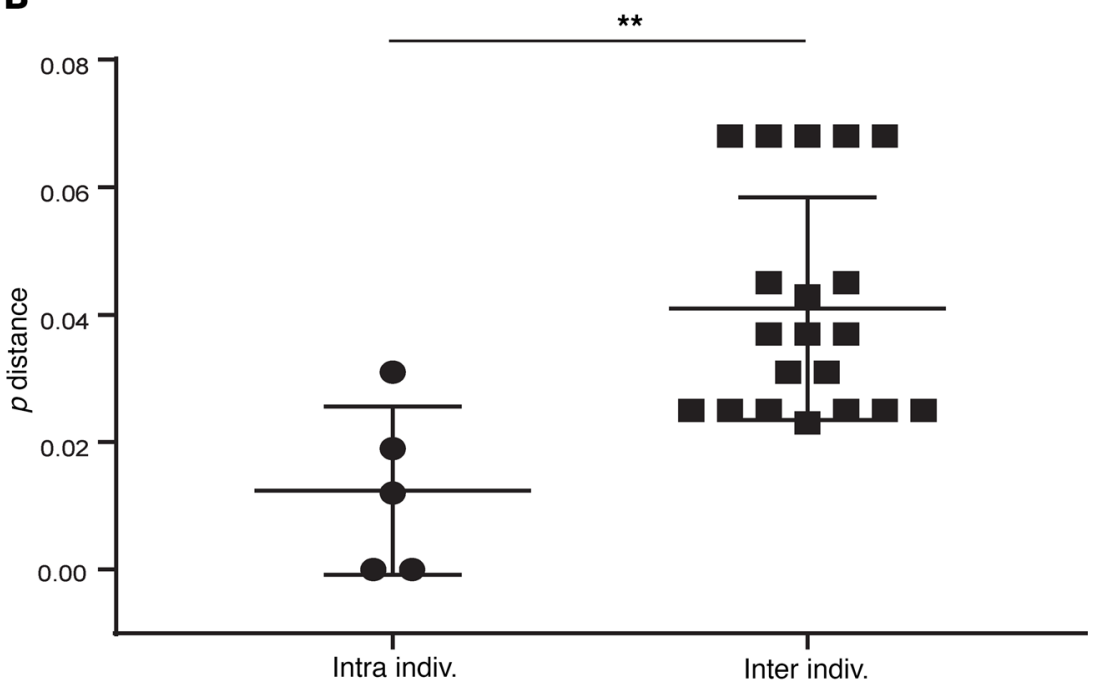

Figure 4. HIV-1 pol sequence analysis reveals that liver macrophages and bulk liver contain genetically similar viruses. $p$ distances between HIV-1 pol amplicons (160 bp) from purified LMs after culture or from bulk liver tissue were calculated. All comparisons were of 160 nucleotide amplicons, except comparisons including subject LT01, which used 44 nucleotide amplicons as that was the largest obtainable sequence. The LTO2 LM sequence included in the analysis was amplified from RNA. (A) Pairwise $p$ distances. Dark red indicates lower values (i.e., more genetically similar), and dark blue indicates higher values (i.e., more genetically distinct). (B) Comparison of inter- and intraindividual $p$ distances between LMs and bulk liver amplicons from the same donor to $p$ distances between LMs and bulk liver amplicons from different individuals. Error bars represent mean $\pm \mathrm{SD}$. $P$ value was calculated by 2-tailed Mann Whitney $U$ test, ${ }^{*} P<0.005$.

Removal of contaminating T cells. As part of the isolation protocol, nonadherent cells were removed from LMs isolated from HIV-infected livers to ensure purity. Following the removal of nonadherent cells, the cultures were treated with Resimmune (donated by Angimmune LLC, Rockville, Maryland). Resimmune is a high-affinity anti-CD3 recombinant diphtheria immunotoxin antibody that combines the Fv portion of a high-avidity anti-CD3 antibody with diphtheria toxin (9). Resimmune has been used to successfully deplete $\mathrm{CD}^{+}$T cells by $99 \%-99.9 \%$ in vivo. We tested the efficacy of $\mathrm{T}$ cell depletion using Resimmune on uninfected $\mathrm{T}$ cells, and also in parallel on monocytederived macrophages to test toxicity (Supplemental Figure 1, C and D).

Excluding contaminating and/or phagocytosed $T$ cells. T cell purity was measured by qPCR. The presence of $\mathrm{T}$ cells in the isolated LMs was

Primary human LMs. To study their role in vivo, LMs were isolated from fresh liver tissues obtained from both NDRI and from the liver transplantation unit at JHH as mentioned above. LMs from fresh liver tissues were isolated as previously described (36). Briefly, 50-g pieces of liver were sectioned and diced into approximately $2-\mathrm{mm}^{3}$ pieces before enzymatic digestion with pronase. The digest was then filtered and subjected to gradient centrifugation after being layered on $16 \%$ histodenz (Sigma Aldrich). The interface was collected, treated with FcR blocking reagent (Miltenyi Biotec, catalog 130059-901), and then depleted of $\mathrm{CD}^{+} \mathrm{T}$ cells using CD3 microbeads (Miltenyi Biotec, catalog 130-050-101). The unstained cells were plated and nonadherent cells were washed after 2 hours, leaving behind adherent LMs. A second isolation procedure was attempted in which the collected cells were again passed through a MACS cell separation column (Miltenyi Biotec) after labeling them with CD14 microbeads (Miltenyi Biotec, catalog 130-050-201); however, CD14 ${ }^{+}$ selection demonstrated lower purity than plastic adherence. Therefore, the plate adherence method was used for all liver tissues presented in this manuscript.

To confirm LM longevity in the presence of HIV-1 infection, LMs that had purity greater than $90 \%$ were purchased from Life Technologies (catalog HUKCCS). These cells were maintained in collagen-1-coated plates (Life Technologies, catalog A11428-03). detected using a sensitive $\mathrm{qPCR}$ for $C D 3 \varepsilon$ mRNA. To determine the lower limit of detection of the qPCR, T cells were isolated from leucopaks using MACS cell separation columns (Miltenyi Biotec). The leucopaks were obtained from individuals who were HIV-1 seronegative. Following isolation, total $\mathrm{T}$ cells were serially diluted to obtain single cells. The isolated RNA was then subjected to cDNA synthesis and qPCR. qPCR for $C D 3 \varepsilon$ mRNA was determined to be more sensitive than a primer/probe set for the recombined TCRs that have been used to assess low-level $\mathrm{T}$ cell contamination or phagocytosis (Supplemental Figure 1A) (8). qPCR CD3 $\varepsilon$ mRNA was also assessed for tissue inhibition (Supplemental Figure 1B). Attempts to characterize adherent macrophages by flow cytometry depleted cells to the point that they were rendered inadequate for subsequent biologic assays.

$L M$ viral outgrowth assays. VOAs were modified and performed to enhance detection of infectious HIV-1 in LMs by inducing production of HIV-1 from infected cells (37). In the absence of a clear standard, LMs were activated using a variety of stimulants to identify the best inducers of virus production using IFNG, IL-4 (Miltenyi Biotec, catalog 130-093-920), IL-13 (EMD Millipore, IL012), zolendronic acid (Sigma-Aldrich, catalog SML0223), recombinant HIV-1 tat clade-B (ProSpec, catalog hiv-129), lipopolysaccharide (Sigma-Aldrich, catalog L5293), and poly I:C (Invivogen, catalog tlrl-piclv) (38-43). The optimal and most consistent induction in vitro was obtained using 2 
A

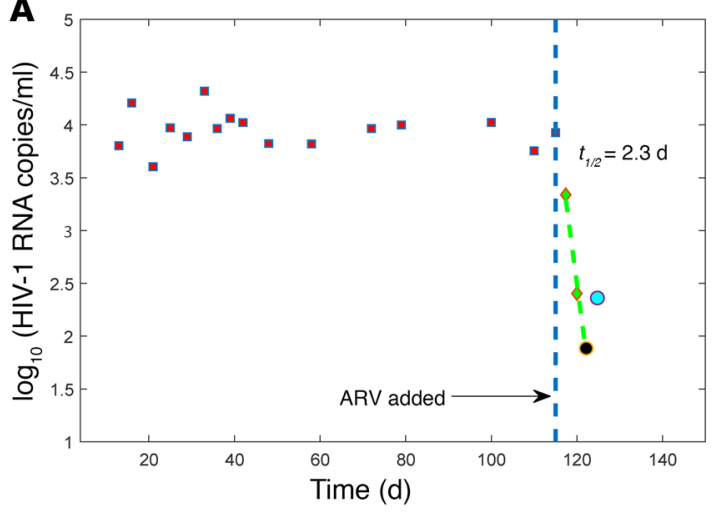

C

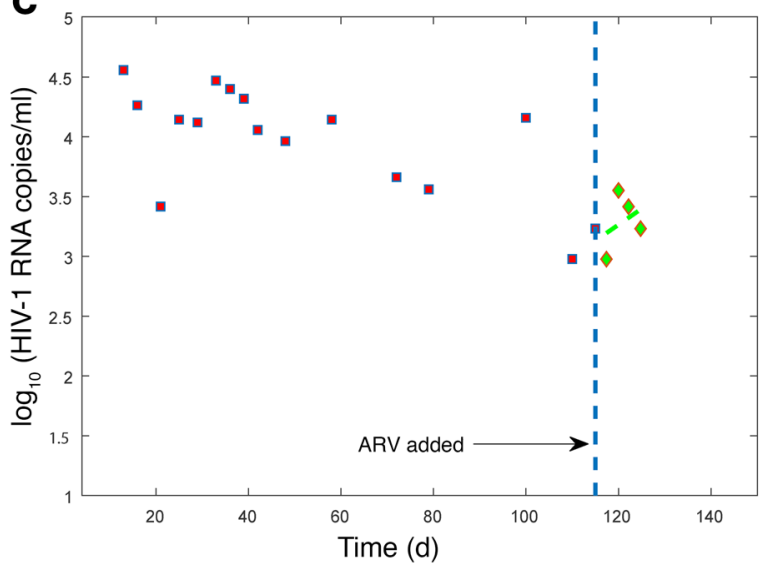

B

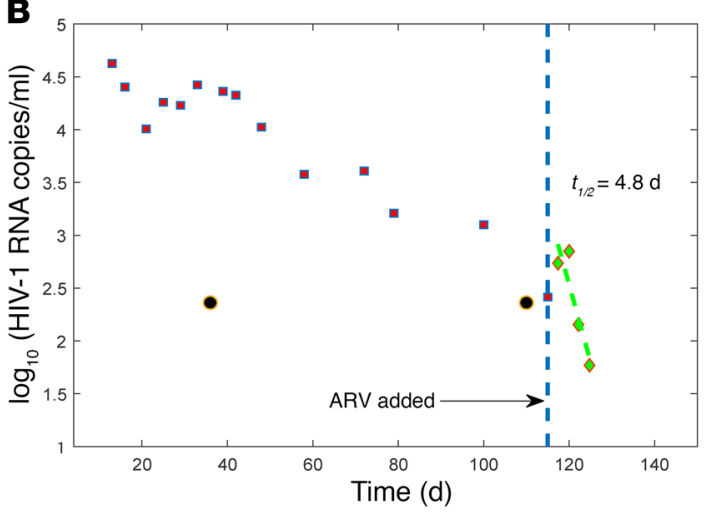

D

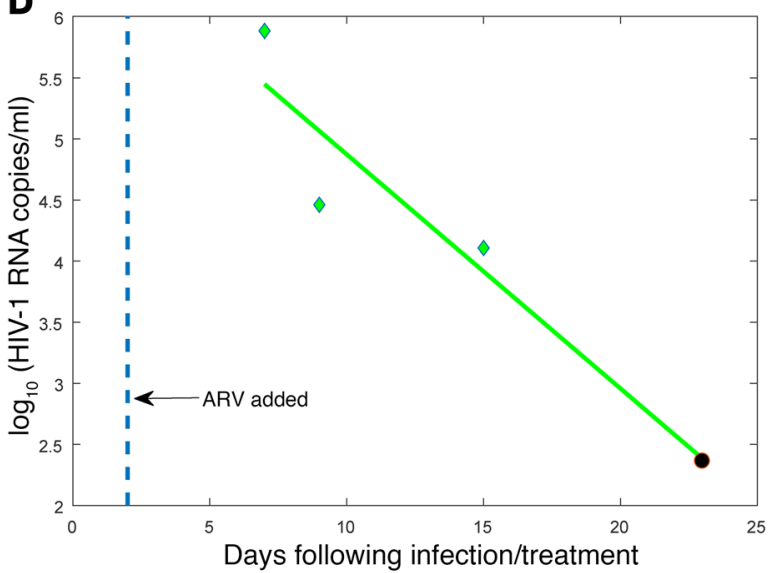

Figure 5. HIV-1-infected LMs persist and decay with inconstant dynamics in vitro. LMs from 3 donors were infected with GFP-HIV-1 and maintained in culture for 100-200 days. HIV-1 RNA was quantified in supernatants during 2 phases: pre-ART and during ART. ART comprised tenofovir disoproxil fumarate $(10 \mu \mathrm{M})$, emtricitabine $(1 \mu \mathrm{M})$, and raltegravir $(1 \mu \mathrm{M})$, all administered at concentrations at least 4-fold higher than their published IC ${ }_{50}$. In parallel, decay in HIV-1 RNA was quantified in HIV-1-infected CD4+ $T$ cells using the same concentrations of ART over the same time course as the second phase of LM infection. Shown are 4 representative panels of decay rates in HIV-1 RNA in supernatants from LMs (A-C) and CD4+ T cells (D). Individual points are plotted before (red) and during (green) ART. The vertical dashed lines indicate when ART was added. Trend lines (green dashes) are indicated, computed using scaling to account for differing intervals between media changes (see Supplemental Material). Moderate declines in HIV-1 RNA were observed in all LM donors pre-ART. Decay of HIV-1-infected LMs can be inferred from supernatant HIV-1 RNA levels during treatment with ART: (A) fast; (B) intermediate; and (C) slow/indefinite decay were all observed in vitro. The $t_{1 / 2}$ was unable to be calculated for wells in which slow/indefinite decay was noted. GFP+ ${ }^{+}$cells were evident in all wells for which HIV-1 RNA was detectable in supernatants.

successive doses of IFNG $(200 \mathrm{U} / \mathrm{ml}) 48$ hours apart followed by 200 ng HIV-1 rTat on 3 consecutive days. rTat was added to the media 48 hours after the second IFNG dose. The VOA for LMs isolated from LT01 was distinct and involved stimulation with IFNG followed by treatment with TLR agonists. All LMs were maintained in the presence of antiretrovirals to prevent their ex vivo infection upon virus release by dying or phagocytosed $\mathrm{T}$ cells.

Following activation, LM supernatants were filtered and transferred to target cells and LMs were cocultured with target cells. To augment viral replication, LM supernatants were transferred every 10-11 days to separate batches of uninfected target cells, whereas LM target cell cocultures were replenished with uninfected target cells repeatedly. LMs and target cell cocultures were maintained for 1 month after initial stimulation. The target cells used in the VOA were either activated $\mathrm{CD}^{+}{ }^{+} \mathrm{T}$ (for LTO1) or CEMx174 cells (ATCC, cata$\log$ CRL-1992) to amplify detection of macrophage-released virus. CEMx174 cells were obtained from Lucio Gama (Johns Hopkins University, Baltimore, Maryland); this cell line is not listed in the database of commonly misidentified cells maintained by the International Cell
Line Authentication Committee (ICLAC). The CEMx174 cell line was remarkably effective for propagating HIV-1 from LMs in our hands in spite of reports that indicate their lack of CCR5, the coreceptor that is commonly utilized by macrophage-tropic HIV-1 virions (Supplemental Figures 3 and 5). In addition, CEMx174 cells have been used to propagate HIV-1 in a macrophage outgrowth assay (44).

Quantitative VOA. The quantitative VOA (QVOA) on resting memory $\mathrm{CD}^{+} \mathrm{T}$ cells (Supplemental Table 1 ) was performed as previously described (15).

HIV-1 infections. To study viral kinetics in LMs, cells were inoculated with the GFP-tagged R5-tropic HIV-1 strain pSF162R3 Nef', which has been previously described as leading to robust infection in MDMs (13). The pSF162R3 Nef ${ }^{+}$was obtained from Amanda Brown (Johns Hopkins University, Baltimore, Maryland); the bicistronic reporter virus had GFP inserted in place of the nefgene and nef added under the control of a separate IRES. The concentration of the viral stock was determined using PCR as described (14). When required, a validated ELISA (Perkin Elmer, catalog NEK050001KT) was used to detect p24. To initiate in vitro infection, a viral titer of $1.10 \times 10^{9}$ copies (p24: $24.86 \mathrm{ng} / \mathrm{ml}$ ) 
and $3.67 \times 10^{8}$ copies ( $24: 74.58 \mathrm{ng} / \mathrm{ml}$ ) of HIV-1 was used for 24 - and 96-well culture plates, respectively. LMs were overlaid with virus for 24 hours, after which they were washed with PBS. HIV-1-uninfected wells from each donor were used as negative controls.

$\mathrm{CD} 4^{+} \mathrm{T}$ cells were isolated from leucopaks derived from HIV-1uninfected individuals using MACS cell separation columns (Miltenyi Biotec). Leucopaks were activated with phytohaemagglutinin (PHA) $(10 \mu \mathrm{g} / \mathrm{ml})$ for 72 hours before isolation of $\mathrm{CD}^{+} \mathrm{T}$ cells. Isolated cells were infected using $\mathrm{HIV}_{-1}$, an X4-tropic strain, at a p24 concentration of $73 \mathrm{ng} / \mathrm{ml}$. CEMx174 cells were also used in VOA, as indicated above. $\mathrm{CD} 4^{+} \mathrm{T}$ cells and CEMx174 cells were incubated with virus by spinoculation at $1,200 \mathrm{~g}, 20^{\circ} \mathrm{C}$ for 2 hours.

Nucleic acid purification and quantification. To determine HIV-1 DNA levels, the DNeasy Blood and Tissue kit was used (Qiagen, catalog 69504). HIV-1 proviral DNA was detected using a modification of a previously described assay for RNA and was not inhibited by cells (Supplemental Figure 6) (45).

The QIAmp viral RNA mini kit (Qiagen, catalog 52906) was used for RNA extraction from supernatants of ex vivo infections and VOAs. To enhance the likelihood of detection of HIV-1 RNA in the VOA for LTO2, CEMx174 supernatants were pooled and ultracentrifuged before RNA extraction. Supernatant HIV-1 RNA titers were determined as described (14). The lower limit of detection of HIV-1 RNA PCR was determined using HIV-1 Acrometrix standards (Life Technologies, catalog 942013).

Phylogenetic analysis. Pol amplicons were aligned and pairwise $p$ distances between amplicons were calculated in Bioedit, version 7.2.5. All comparisons were of 162 nucleotide amplicons, except comparisons including subject LT01, which used 44 nucleotide amplicons. Mixed bases present at some positions in some amplicons were considered a single nucleotide change in the analysis.

Antiretroviral therapy. After isolation of LMs from HIV-1-infected people on ART, efforts were taken to prevent LMs from being infected ex vivo, so that results would only be consistent with in vivo LM infection. To prevent ex vivo HIV-1 infection of LMs, bulk liver tissue and isolated LMs were maintained in media containing tenofovir disoproxil fumarate $(10 \mu \mathrm{M})$, emtricitabine $(1 \mu \mathrm{M})$, and raltegravir $(1 \mu \mathrm{M})$ until the VOA was initiated. Antiretrovirals were also used to observe the effect of treatment on in vitro infections of LMs using previously described concentrations (46-49). In the control (uninfected wells), antiretrovirals were not toxic to LMs.

Modeling the half-life of LMs. A standard viral dynamic model of HIV-1 infection (50) was adapted for this cell culture system as described in the Supplemental Material.

Statistics. The 2-tailed Mann-Whitney $U$ test was used to calculate the $P$ value to determine the significance of inter- and intraindividual $p$ distances between LMs and bulk liver amplicons from the same donor to $p$ distances between LMs and bulk liver amplicons from different individuals. $P<0.005$ was considered significant.

Study approval. This study protocol was approved by the Johns Hopkins School of Medicine institutional review board, and was considered exempt from human subject research because all samples were obtained strictly for clinical reasons or postmortem, and would otherwise have been discarded. Hence, it was determined that informed consent was not required.

\section{Author contributions}

$\mathrm{AJK}, \mathrm{SS}$, JS, and JQ conducted the experiments. AJK and AB designed and analyzed the data. JRB assisted in sequence analysis. CMD assisted with editing of the manuscript. CMD and AMC procured liver explants. AG and ASP developed the mathematical models of in vitro data. $\mathrm{AJK}$ and $\mathrm{AB}$ wrote the manuscript. $\mathrm{AB}$ supervised the project.

\section{Acknowledgments}

We would like to thank Robert F. Siliciano, Deborah Persaud, and David L. Thomas for helpful discussions and critical review of our manuscript. Angimmune LLC donated Resimmune. We thank Amanda Brown for the bicistronic GFP reporter virus (pSF162R3 $\mathrm{Nef}^{+}$) and Lucio Gama for CEMx174 cells. This study was supported by NIH grants K08 AI 081544, R01 DA 016078, R56 AI 118445, and amfAR grant 108814-55-RGRL to AB; amfAR grant 108707-54-RKRL to CMD; and NIH grants R01 AI024333, R01 OD 011095, R01 AI116868, and P01 AI131365 to ASP.

Address correspondence to: Ashwin Balagopal, 855 N. Wolfe Street, Baltimore, Maryland 21205, USA. Phone: 443.287.7666; Email: abalago1@jhmi.edu.
1. Hütter G, et al. Long-term control of HIV by CCR5 Delta32/Delta32 stem-cell transplantation. N Engl J Med. 2009;360(7):692-698.

2. Siliciano RF, Greene WC. HIV latency. Cold Spring Harb Perspect Med. 2011;1(1):a007096.

3. Kandathil AJ, Sugawara S, Balagopal A. Are T cells the only HIV-1 reservoir? Retrovirology. 2016;13(1):86.

4. Honeycutt JB, et al. Macrophages sustain HIV replication in vivo independently of T cells. JClin Invest. 2016;126(4):1353-1366.

5. Honeycutt JB, et al. HIV persistence in tissue macrophages of humanized myeloid-only mice during antiretroviral therapy. Nat Med. 2017;23(5):638-643.

6. DiNapoli SR, et al. Tissue-resident macrophages can contain replication-competent virus in antiretroviral-naive, SIV-infected Asian macaques. JCI Insight. 2017;2(4):e91214.

7. Jenne $\mathrm{CN}$, Kubes P. Immune surveillance by the liver. Nat Immunol. 2013;14(10):996-1006.
8. Calantone N, et al. Tissue myeloid cells in SIV-infected primates acquire viral DNA through phagocytosis of infected T cells. Immunity. 2014;41(3):493-502.

9. Woo JH, et al. GMP production and characterization of the bivalent anti-human T cell immunotoxin, A-dmDT390-bisFv(UCHT1) for phase I/II clinical trials. Protein Expr Purif. 2008;58(1):1-11.

10. Frankel AE, et al. Anti-CD3 recombinant diphtheria immunotoxin therapy of cutaneous T cell lymphoma. Curr Drug Targets. 2009;10(2):104-109.

11. Laird GM, et al. Rapid quantification of the latent reservoir for HIV-1 using a viral outgrowth assay. PLoS Pathog. 2013;9(5):e1003398.

12. Avalos CR, et al. Brain macrophages in simian immunodeficiency virus-infected, antiretroviralsuppressed macaques: a functional latent reservoir. MBio. 2017;8(4):e01186-17.

13. Brown AM. Use of a macrophage-tropic GFPtagged human immunodeficiency virus type 1
(HIV-1) to study viral reservoirs. Methods Mol Biol. 2009;515:165-175.

14. Shan L, et al. A novel PCR assay for quantification of HIV-1 RNA. J Virol. 2013;87(11):6521-6525.

15. Durand CM, et al. HIV-1 DNA is detected in bone marrow populations containing CD4+ T cells but is not found in purified CD34+ hematopoietic progenitor cells in most patients on antiretroviral therapy. JInfect Dis. 2012;205(6):1014-1018.

16. Stevenson M. HIV-1 pathogenesis. Nat Med. 2003;9(7):853-860.

17. Sattentau QJ, Stevenson M. Macrophages and HIV-1: an unhealthy constellation. Cell Host Microbe. 2016;19(3):304-310.

18. Epelman S, Lavine KJ, Randolph GJ. Origin and functions of tissue macrophages. Immunity. 2014;41(1):21-35.

19. Ginhoux F, et al. Fate mapping analysis reveals that adult microglia derive from primitive macrophages. Science. 2010;330(6005):841-845.

20. Hashimoto D, et al. Tissue-resident macrophages 
self-maintain locally throughout adult life with minimal contribution from circulating monocytes. Immunity. 2013;38(4):792-804.

21. Klein I, et al. Kupffer cell heterogeneity: functional properties of bone marrow derived and sessile hepatic macrophages. Blood. 2007;110(12):4077-4085.

22. Schulz C, et al. A lineage of myeloid cells independent of Myb and hematopoietic stem cells. Science. 2012;336(6077):86-90.

23. Yona S, et al. Fate mapping reveals origins and dynamics of monocytes and tissue macrophages under homeostasis. Immunity. 2013;38(1):79-91.

24. Bouwens L, Baekeland M, De Zanger R, Wisse E. Quantitation, tissue distribution and proliferation kinetics of Kupffer cells in normal rat liver. Hepatology. 1986;6(4):718-722.

25. Cao YZ, Dieterich D, Thomas PA, Huang YX, Mirabile M, Ho DD. Identification and quantitation of HIV-1 in the liver of patients with AIDS. AIDS. 1992;6(1):65-70.

26. Housset C, et al. Presence of HIV-1 in human parenchymal and non-parenchymal liver cells in vivo. J Hepatol. 1993;19(2):252-258.

27. Schmitt MP, et al. Permissivity of primary cultures of human Kupffer cells for HIV-1. AIDS Res Hum Retroviruses. 1990;6(8):987-991.

28. Schmitt MP, et al. Multiplication of human immunodeficiency virus in primary cultures of human Kupffer cells-possible role of liver macrophage infection in the physiopathology of AIDS. Res Virol. 1990;141(2):143-152.

29. Schweitzer C, et al. Morphine stimulates HIV replication in primary cultures of human Kupffer cells. Res Virol. 1991;142(2-3):189-195.

30. St Gelais C, Wu L. SAMHD1: a new insight into HIV-1 restriction in myeloid cells. Retrovirology. 2011;8:55.

31. Tada T, et al. MARCH8 inhibits HIV-1 infection by reducing virion incorporation of envelope glycoproteins. Nat Med. 2015;21(12):1502-1507.

32. Pollack RA, et al. Defective HIV-1 proviruses are expressed and can be recognized by cytotoxic T lymphocytes, which shape the proviral landscape. Cell Host Microbe. 2017;21(4):494-506.e4.

33. Igarashi $\mathrm{T}$, et al. Macrophage are the principal reservoir and sustain high virus loads in rhesus macaques after the depletion of $\mathrm{CD} 4+\mathrm{T}$ cells by a highly pathogenic simian immunodeficiency virus/HIV type 1 chimera (SHIV): implications for HIV-1 infections of humans. Proc Natl Acad Sci US A. 2001;98(2):658-663.

34. Clements JE, et al. The central nervous system is a viral reservoir in simian immunodeficiency virusinfected macaques on combined antiretroviral therapy: a model for human immunodeficiency virus patients on highly active antiretroviral therapy. JNeurovirol. 2005;11(2):180-189.

35. Eriksson S, et al. Comparative analysis of measures of viral reservoirs in HIV-1 eradication studies. PLoS Pathog. 2013;9(2):e1003174.

36. Alabraba EB, Curbishley SM, Lai WK, Wigmore SJ, Adams DH, Afford SC. A new approach to isolation and culture of human Kupffer cells. J Immunol Methods. 2007;326(1-2):139-144.

37. Siliciano JD, Siliciano RF. Enhanced culture assay for detection and quantitation of latently infected, resting CD4+ T-cells carrying replicationcompetent virus in HIV-1-infected individuals. Methods Mol Biol. 2005;304:3-15.

38. Brown A, Zhang H, Lopez P, Pardo CA, Gartner S In vitro modeling of the HIV-macrophage reservoir. J Leukoc Biol. 2006;80(5):1127-1135.

39. Su X, et al. Interferon- $\gamma$ regulates cellular metabolism and mRNA translation to potentiate macrophage activation. Nat Immunol. 2015;16(8):838-849.

40. Karn J. The molecular biology of HIV latency: breaking and restoring the Tat-dependent transcriptional circuit. Curr Opin HIV AIDS.
2011;6(1):4-11.

41. Caron NJ, et al. Intracellular delivery of a Tat-eGFP fusion protein into muscle cells. Mol Ther. 2001;3(3):310-318.

42. Mosser DM, Edwards JP. Exploring the full spectrum of macrophage activation. Nat Rev Immunol. 2008;8(12):958-969.

43. Donahue DA, Kuhl BD, Sloan RD, Wainberg MA. The viral protein Tat can inhibit the establishment of HIV-1 latency. J Virol. 2012;86(6):3253-3263.

44. Avalos CR, et al. Quantitation of productively infected monocytes and macrophages of simian immunodeficiency virus-infected macaques. JVirol. 2016;90(12):5643-5656.

45. Müller J, et al. A novel internally controlled realtime reverse transcription-PCR assay for HIV-1 RNA targeting the pol integrase genomic region. J Virol Methods. 2007;142(1-2):127-135.

46. Gavegnano C, Detorio MA, Bassit L, Hurwitz SJ, North TW, Schinazi RF. Cellular pharmacology and potency of HIV-1 nucleoside analogs in primary human macrophages. Antimicrob Agents Chemother. 2013;57(3):1262-1269.

47. Hazen R, Lanier ER. Relative anti-HIV-1 efficacy of lamivudine and emtricitabine in vitro is dependent on cell type. J Acquir Immune Defic Syndr. 2003;32(3):255-258.

48. Iwamoto M, et al. Safety, tolerability, and pharmacokinetics of raltegravir after single and multiple doses in healthy subjects. Clin Pharmacol Ther. 2008;83(2):293-299.

49. Scopelliti F, et al. Comparative antiviral activity of integrase inhibitors in human monocytederived macrophages and lymphocytes. Antiviral Res. 2011;92(2):255-261.

50. Perelson AS, Neumann AU, Markowitz M, Leonard JM, Ho DD. HIV-1 dynamics in vivo: virion clearance rate, infected cell life-span, and viral generation time. Science. 1996;271(5255):1582-1586. 